\title{
Mortality Caused by Late-onset Sepsis in Very Low Birth Weight Infants: Risk Analysis and the Performance of Diagnostic Tools
}

\author{
Hakan Ongun ${ }^{1}$ and Meltem Demir ${ }^{2}$ \\ ${ }^{1}$ Division of Neonatology, Department of Pediatrics, Faculty of Medicine, Istinye University Antalya Medical Park Hospital, Antalya, \\ Turkey \\ ${ }^{2}$ Department of Medical Biochemistry, Faculty of Medicine, İstinye University, Antalya, Turkey
}

\begin{abstract}
Objective: To assess the risk on late-onset sepsis attributed mortality in very low birth weight (VLBW) infants.

Study Design: Observational study.

Place and Duration of Study: Level-III Neonatal Intensive Care Unit, İstinye University, Antalya Medical Park Hospital, Turkey, between January 2014 and December 2018.

Methodology: Perinatal characteristics and clinical features of 198 septic preterm neonates were evaluated to predict sepsisattributed mortality. ROC analysis was employed to drive optimal-cutoffs for laboratory parameters and logistic regression to calculate mortality risk factors using SPSS version-22 and MedCalc software.

Results: Mean gestational age was $28.91 \pm 2.67$ weeks. Umbilical catheterisation was the principal risk factor for culture-positive sepsis (OR $2.860,95 \% \mathrm{Cl}: 1.232-6.639)$. Outborn infants were more likely to deliver surfactant and longer intubation $(p=0.013$, and $p=0.005$, respectively), manifested frequent BPD $(p=0.014)$, and at greater risk of proven sepsis and mortality (OR: 1.796, 95\% Cl: 1.011-3.191; OR: 1.950, 95\%Cl: 1.002-3.794). Low Apgar scores necrotising enterocolitis (NEC) and prolonged intubation were independent risk factors for mortality (OR: $13.840,95 \% \mathrm{Cl}: 6.384-30.005$; OR: $5.410,95 \% \mathrm{Cl}$ : 2.113-13.849; OR: $10.037,95 \% \mathrm{Cl}: 4.700-21.434)$. An increase in high-sensitivity C-reactive protein (hsCRP)-ratio $>6.08$-fold afforded good sensitivity and specificity (AUC: 0.914 ; sensitivity: $89.36 \%$, specificity: $86.09 \%$ ). Logistic regression of various combinations has shown a $>6.08$-fold change in hsCRP-ratio over 24 -hours and platelet counts $<88 \times 10^{9} / \mathrm{L}$ optimally predicted mortality (OR: $27.983,95 \% \mathrm{Cl}$ : 9.704-80.697).

Conclusion: Low Apgar scores, NEC and prolonged intubation are independent risk factors for mortality of VLBW infants. Birth in level III-IV NICUs featuring special neonatal care, avoidance of prolonged intubation, and timely prediction of fatal sepsis using hsCRP ratio and platelets could prevent sepsis-related mortality.
\end{abstract}

Key Words: Sepsis, Very low birth weight, Infant formality, Apgar score.

How to cite this article: Ongun H, Demir M. Mortality Caused by Late-onset Sepsis in Very Low Birth Weight Infants: Risk Analysis and the Performance of Diagnostic Tools. J Coll Physicians Surg Pak 2020; 30(06):611-616 https://doi.org/10.29271/jcpsp.2020.06.611.

\section{INTRODUCTION}

Late-onset sepsis (LOS) is one of the most devastating problems encountered in neonatal intensive care units (NICUs). ${ }^{1}$ The incidence ranges from 5 to $29.4 \%$ in very low birth weight (VLBW) infants, 10 -fold that of theirterm-born counterparts. ${ }^{2,3}$ Although the incidence of LOS has declined modestly, LOS remains a lifethreatening problem in both high-and low-income countries. ${ }^{4}$

Correspondence to: Hakan Ongun, Faculty of Medicine, Istinye University, Antalya Medical Park Hospital, Tekelioglu Caddesi No. 7, Muratpasa, Antalya, Turkey

E-mail: hongun88@hotmail.com

Received: April 15, 2020; Revised: June 13, 2020;

Accepted: June 26, 2020

DOI: https://doi.org/10.29271/jcpsp.2020.06.611
VLBW infants are at increased risk of invasive infections because their neutrophil functions are poor and their maternally-acquired immunoglobulin levels low..$^{3-6}$ Early recognition of a septic infant and timely antibiotic therapy are key factors to determine mortality. ${ }^{7}$ Unfortunately, the subtle and indistinct clinical symptoms cause diagnostic delays. Although several predictive models and aids to diagnosis (e.g. cytokine levels) have been developed, the reference values vary by gestational age (GA) and postnatal age, rendering interpretation challenging. ${ }^{3,8}$ The associated financial costs limit the utility of specific markers in low-to middle-income countries. ${ }^{3}$ It is essential to identify a deteriorating septic infant using routine laboratory parameters. The objective of this observational cohort study of VLBW infants, was to determine the risk for LOS mortality and assess the predictive utility of sepsis work-up tools commonly used in daily practice. 
Table I: Demographics and risk factors for mortality.

\begin{tabular}{|c|c|c|c|c|}
\hline Demographics & $\begin{array}{l}\text { Non-survivors } \\
\quad(n=47)\end{array}$ & $\begin{array}{l}\text { Survivors } \\
(n=151)\end{array}$ & $\begin{array}{l}\text { Odds ratio } \\
(95 \% \mathrm{Cl})\end{array}$ & p-value \\
\hline Gestational age, (weeks) (mean \pm SD) & $27.38 \pm 2.79$ & $29.39 \pm 2.46$ & $0.745(0.651-0.853)$ & $<0.001$ \\
\hline Birth weight, (grams) (mean $\pm S D$ ) & $1020.26 \pm 410.06$ & $1308.59 \pm 428.62$ & $0.998(0.997-0.999)$ & $<0.001$ \\
\hline Male gender, (\%) & $30(63.8 \%)$ & $75(49.7 \%)$ & $1.788(0.910-3.513)$ & 0.092 \\
\hline Maternal age, (years) (mean \pm SD) & $28.53 \pm 5.90$ & $29.48 \pm 6.52$ & $0.976(0.927-1.029)$ & 0.371 \\
\hline Maternal condition, $(\%)$ & $10(21.3 \%)$ & $47(31.1 \%)$ & $0.598(0.274-1.303)$ & 0.196 \\
\hline Preeclampsia, (\%) & $5(10.6 \%)$ & $33(21.9 \%)$ & $0.426(0.156-1.162)$ & 0.096 \\
\hline Gestational diabetes mellitus, (\%) & $4(8.5 \%)$ & $7(4.6 \%)$ & $1.914(0.535-6.847)$ & 0.318 \\
\hline Twins, (\%) & $10(21.3 \%)$ & $52(34.4 \%)$ & $0.515(0.237-1.117)$ & 0.093 \\
\hline Outborn infant, (\%) & $28(59.6 \%)$ & $65(43 \%)$ & $1.950(1.002-3.794)$ & 0.049 \\
\hline Intrauterine transport, (\%) & $10(21.3 \%)$ & $10(6.6 \%)$ & $3.811(1.476-9.837)$ & 0.006 \\
\hline CS delivery, (\%) & $38(80.9 \%)$ & $134(88.7 \%)$ & $1.867(0.771-4.522)$ & 0.162 \\
\hline PROM, (\%) & $2(4.3 \%)$ & $6(4.0 \%)$ & $1.074(0.209-5.509)$ & 0.932 \\
\hline \multicolumn{5}{|l|}{ Risk factors } \\
\hline 5. Minutes Apgar score <7, (\%) & $34(72.3 \%)$ & $24(15.9 \%)$ & $13.840(6.384-30.005)$ & $<0.001$ \\
\hline Surfactant administration, (\%) & $46(97.9 \%)$ & $90(59.6 \%)$ & $31.178(4.187-232.142)$ & 0.001 \\
\hline Lenghth of intubation $>5$ days $a,(\%)$ & $35(74.5 \%)$ & $34(22.5 \%)$ & $10.037(4.700-21.434)$ & $<0.001$ \\
\hline CPAP therapy & $1(2.1 \%)$ & $127(84.1 \%)$ & $0.004(0.001-0.031)$ & $<0.001$ \\
\hline Bronchopulmonary dsyplasia, (\%) & $0(0 \%)$ & $19(12.6 \%)$ & - & NA \\
\hline VP shunt, (\%) & $1(2.1 \%)$ & $5(3.3 \%)$ & $0.635(0.072-5.573)$ & 0.682 \\
\hline PDA, (\%) & $16(34.0 \%)$ & $52(34.4 \%)$ & $0.983(0.493-1.960)$ & 0.960 \\
\hline NEC, (\%) & $12(25.5 \%)$ & $9(6 \%)$ & $5.410(2.113-13.849)$ & $<0.001$ \\
\hline Umbilical catheter, (\%) & $47(100 \%)$ & $114(75.5 \%)$ & - & NA \\
\hline $\begin{array}{l}\text { Peripherally-inserted central venous } \\
\text { catheter, (\%) }\end{array}$ & $24(51.1 \%)$ & $24(15.9 \%)$ & $5.522(2.690-11.336)$ & $<0.001$ \\
\hline Antacid medication, (\%) & $18(38.3 \%)$ & $0(0 \%)$ & - & NA \\
\hline Urine catheter, (\%) & $0(0 \%)$ & $3(2 \%)$ & - & NA \\
\hline Culture positivity, (\%) & $20(42.6 \%)$ & $59(39.1 \%)$ & $1.155(0.594-2.244)$ & 0.671 \\
\hline Coagulase (-) staphylococcus & $8(17.0 \%)$ & $19(12.6 \%)$ & $1.425(0.579-3.505)$ & 0.440 \\
\hline Enterococcus* & $1(2.1 \%)$ & $17(11.3 \%)$ & $0.171(0.022-1.324)$ & 0.091 \\
\hline Gram (-) bacteria ** & $8(17.0 \%)$ & $23(15.2 \%)$ & $1.142(0.473-2.754)$ & 0.768 \\
\hline Fungi & $3(6.4 \%)$ & $0(0 \%)$ & - & NA \\
\hline
\end{tabular}

\section{METHODOLOGY}

This observational study was approved by the Institutional Review Board (approval No. 2019/3, dated April 23, 2019).

The medical records of NICU admissions with ICD-codes of 'neonatal sepsis between January 2014 and December 2018 were retrospectively extracted from the hospital patient database. All preterm infants of $\mathrm{GA}<32$ weeks and birth weight $<1,500 \mathrm{~g}$, with at least one episode of LOS (sepsis after 3 days of life) were included. Infants with early-onset sepsis, intraventricular haemorrhage and/or who underwent any surgical inter- vention prior to sepsis suspicion were excluded because the laboratory data might be affected by inflammation.

Pre- and ante-natal data included; birthplace (outborn: born elsewhere and then transferred to the study unit); clinical sepsis signs (apnea, increased oxygen-dependence, feeding intolerance, skin mottling, tachycardia, impaired peripheral perfusion and mental status, lethargy, seizures, temperature instability, and unexplained acidosis); ${ }^{9}$ and potential sepsis risk factors were extracted from patients' records. If two blood cultures were positive for coagulase-negative (CoNS), it was considered that CoNS caused the sepsis staphylococci. ${ }^{10}$ 
Table II: The performance of diagnostic tools to determine mortality.

\begin{tabular}{|c|c|c|c|c|c|c|c|c|}
\hline & $\begin{array}{c}\text { Cut-off } \\
\text { value }\end{array}$ & $\begin{array}{c}\text { AUC } \\
(95 \% \mathrm{CL})\end{array}$ & $\begin{array}{c}\text { Sensitivity } \\
(95 \% \mathrm{CI})\end{array}$ & $\begin{array}{c}\text { Specificity } \\
(95 \% \mathrm{CI})\end{array}$ & $\begin{array}{c}\text { LH+ } \\
(95 \% \mathrm{Cl})\end{array}$ & $\begin{array}{c}\text { PPV } \\
(95 \% \mathrm{Cl})\end{array}$ & $\begin{array}{c}\text { NPV } \\
(95 \% \mathrm{CI})\end{array}$ & $\mathbf{p}$ \\
\hline \multicolumn{9}{|l|}{ Day 1} \\
\hline $\mathrm{hsCRP}_{\text {dayo }}(\mathrm{mg} / \mathrm{dL})$ & 1.08 & $0.621(0.540-0.703)$ & $72.34(57.4-84.4)$ & $58.28(50.0-66.2)$ & $1.73(1.3-2.2)$ & $35.1(25.6-45.4)$ & $87.1(79.0-93.0)$ & 0.0035 \\
\hline Hematocrit (\%) & 46.8 & $0.509(0.422-0.597)$ & $72.34(57.4-84.4)$ & $39.7(31.9-48.0)$ & $1.20(1.0-1.5)$ & $27.2(19.6-35.9)$ & $82.2(71.5-90.2)$ & 0.838 \\
\hline WBC $\left(\times 10^{9} / L\right)$ & 22.3 & $0.611(0.504-0.718)$ & $42.55(28.3-57.8)$ & $87.42(81.0-92.3)$ & $3.38(2.0-5.8)$ & $51.3(34.8-67.6)$ & $83.0(76.3-88.5)$ & 0.042 \\
\hline Neutrophil count $\left(\times 10^{9} / L\right)$ & 1.6 & $0.528(0.431-0.625)$ & $25.53(13.9-40.3)$ & $83.44(76.5-89.0)$ & $1.54(0.8-2.8)$ & $32.4(18.0-49.8)$ & $78.3(71.1-84.4)$ & 0.570 \\
\hline NLR & 3.66 & $0.508(0.413-0.604)$ & $10.64(3.5-23.1)$ & $96.69(92.4-98.9)$ & $3.21(1.0-10.6)$ & $50.0(18.7-81.3)$ & $77.7(71.0-83.4)$ & 0.865 \\
\hline PLR & 34.57 & $0.502(0.408-0.596)$ & $25.53(13.9-40.3)$ & $63.58(55.4-71.2)$ & $0.70(0.4-1.2)$ & $17.9(9.6-29.2)$ & $73.3(64.8-80.6)$ & 0.966 \\
\hline Platelets count $\left(\times 10^{9} / \mathrm{L}\right)$ & 88 & $0.534(0.437-0.631)$ & $19.15(9.1-33.3)$ & $90.73(84.9-94.8)$ & $2.07(1.0-4.5)$ & $39.1(19.7-61.5)$ & $78.3(71.5-84.2)$ & 0.496 \\
\hline MPV & 9.6 & $0.532(0.435-0.629)$ & $38.30(24.5-53.6)$ & $72.19(64.3-79.2)$ & $1.38(0.9-2.1)$ & $30.0(18.8-43.2)$ & $79.0(71.2-85.5)$ & 0.517 \\
\hline \multicolumn{9}{|l|}{ Day 1} \\
\hline $\mathrm{hsCRP}_{\text {day1 }}(\mathrm{mg} / \mathrm{dL})$ & 7.17 & $0.868(0.815-0.921)$ & $93.62(82.5-98.7)$ & $66.89(58.8-74.3)$ & $2.83(2.2-3.6)$ & $46.8(36.4-57.4)$ & $97.1(91.8-99.4)$ & $<0.001$ \\
\hline Change in hsCRP ratio & 6.08 & $0.914(0.857-0.971)$ & $89.36(76.9-96.5)$ & $86.09(79.5-91.2)$ & $6.43(4.3-9.7)$ & $66.7(53.7-78.0)$ & $96.3(91.6-98.8)$ & $<0.001$ \\
\hline Hematocrit (\%) & 38.7 & $0.507(0.410-0.604)$ & $34.04(20.9-49.3)$ & $72.19(64.3-79.2)$ & $1.22(0.8-2.0)$ & $27.6(16.7-40.9)$ & $77.9(70.1-84.4)$ & 0.889 \\
\hline WBC $\left(\times 10^{9} / L\right)$ & 18.41 & $0.510(0.413-0.607)$ & $44.68(30.2-59.9)$ & $64.90(56.7-72.5)$ & $1.27(0.9-1.9)$ & $28.4(18.5-40.1$ & $79.0(70.8-85.8)$ & 0.837 \\
\hline Neutrophil count $\left(\times 10^{9} / L\right)$ & 8.1 & $0.503(0.409-0.598)$ & $65.96(50.7-79.1)$ & $28.48(21.4-36.4)$ & $0.92(0.7-1.2)$ & $22.3(15.7-30.1)$ & $72.9(59.7-83.6)$ & 0.943 \\
\hline NLR & 0.80 & $0.502(0.407-0.598)$ & $38.30(24.5-53.6)$ & $55.63(47.3-63.7)$ & $0.86(0.6-1.3)$ & $21.2(13.1-31.4)$ & $74.3(65.3-82.1)$ & 0.960 \\
\hline PLR & 34.23 & $0.550(0.449-0.651)$ & $51.06(36.1-65.9)$ & $66.89(58.8-74.3)$ & $1.54(1.1-2.2)$ & $32.4(22.0-44.3)$ & $81.5(73.5-87.9)$ & 0.331 \\
\hline Platelets count $\left(\times 10^{9} / \mathrm{L}\right)$ & 285 & $0.577(0.483-0.670)$ & $72.34(57.4-84.4)$ & $46.36(38.2-54.6)$ & $1.35(1.1-1.7)$ & $29.6(21.4-38.8)$ & $84.3(74.7-91.4)$ & 0.108 \\
\hline MPV & 10.2 & $0.558(0.463-0.654)$ & $51.06(36.1-65.9)$ & $63.58(55.4-71.2)$ & $1.40(1.0-2.0)$ & $30.4(20.5-41.8)$ & $80.7(72.4-87.3)$ & 0.231 \\
\hline
\end{tabular}

Table III: The combination of diagnostic tools for sepsis-attributed mortality.

\begin{tabular}{|c|c|c|c|c|}
\hline & \multirow{2}{*}{$\operatorname{Exp}(B)$} & \multicolumn{2}{|c|}{ 95\% C.I. for EXP (B) } & \multirow{2}{*}{$\mathbf{p}$} \\
\hline & & Lower & Upper & \\
\hline $\mathrm{CRP}_{\text {dayo }}$ & 1.022 & 0.815 & 1.281 & 0.853 \\
\hline $\mathrm{CRP}_{\text {day } 1}$ & 1.173 & 1.119 & 1.229 & $<0.001$ \\
\hline White blood cell count (WBC) dayo & 1.058 & 1.026 & 1.090 & $<0.001$ \\
\hline White blood cell count (WBC) day1 & 1.004 & 0.973 & 1.036 & 0.804 \\
\hline Platelets count $_{\text {day } 0 .}$ & 0.993 & 0.990 & 0.997 & $<0.001$ \\
\hline Platelets count $_{\text {day } 1}$ & 0.998 & 0.996 & 1.000 & 0.084 \\
\hline [Change in hsCrp ratio $>6.08]+\left[\right.$ Platelets $_{\text {day } 0}<88.000 \mathrm{~mm}^{3}$ ] & 27.983 & 9.704 & 80.697 & $<0.001$ \\
\hline [Change in hsCrp ratio $>6.08]+\left[W_{\text {day } 0}>22300 \mathrm{~mm}^{3}\right.$ ] & 18.124 & 6.230 & 52.730 & $<0.001$ \\
\hline [Platelets $\left._{\text {day } 0}<88.000 \mathrm{~mm}^{3}\right]+\left[W B C_{\text {day } 0}>22300 \mathrm{~mm}^{3}\right]$ & 16.914 & 4.529 & 63.172 & $<0.001$ \\
\hline
\end{tabular}

Infants were grouped by blood culture data (proven sepsis: blood culture positivity with clinical deterioration; clinical sepsis: clinical deterioration in the absence of culture positivity) ${ }^{11}$ and survival (survivors and non-survivors). Sepsis work-up included both a complete blood cell count (CBC) and high-sensitivity C-reactive protein CRP (hSCRP) measurements on the day of clinical sepsis suspicion (day 0 ) and 24 hours thereafter (day 1). Hematocrit, white blood cell count (WBC), neutrophil and lymphocyte counts and percentage, the neutrophil/lymphocyte ratio (NLR), the platelet count, the mean platelet volume (MPV), the platelet/lymphocyte ratio (PLR) and the hsCRP level were analysed. A WBC $>20 \times 10^{9} / \mathrm{L}$ evidenced leukocytosis and a $\mathrm{WBC}<4 \times 10^{9} / \mathrm{L}$ leukopenia; ${ }^{12}$ platelets count $<100 \times 10^{9} / \mathrm{L}$ indicated thrombocytopenia. ${ }^{13}$ Changes in hSCRP ratio were recorded between two serial measurements (calculated by the change of CRP level to the level at day 0$){ }^{14}$

$$
\text { (Changes in hsCRP ratio) }=\frac{\left(\text { hsCRP }_{\text {day } 1}-\text { hsCRP }_{\text {day }}\right)}{\text { hsCRP dayo }}
$$


CBCs were obtained using a Sysmex XN-1000 analyser (Symex, Japan). hsCRP measurements were automated using the immunoturbidimetric method of the modular analyser (Roche Diagnostics, Milano, Italy) (detection limit 0.03 $\mathrm{mg} / \mathrm{L})$.

SPSS version 22 software (IBM Corp., Armonk, NY, USA) was employed for statistical analyses. The Chi-squared and Fisher exact tests were used to compare categorical variables; continuous quantitative variables were compared with the aid of the Mann-Whitney U-test (non-normally distributed parameters) or the Student t-test (normally distributed parameters). Results were given as percentages (\%), means \pm standard deviations, or medians with interquartile ranges (IQRs). Receiver operator characteristic (ROC) curves were drawn using MedCalc software (free trial version) and employed to derive optimal cutoffs, sensitivities, specificities, positive predictive values (PPVs), and negative predictive values (NPVs) of parameters associated with mortality. Logistic regression was employed to calculate mortality risk factors. Estimates were expressed as odds ratios (OR) with $95 \%$ confidence intervals $(\mathrm{Cl})$. A p-value $<0.05$ was considered to indicate statistical significance.

\section{RESULTS}

Two hundred and five preterm neonates of 914 NICU admissions were evaluated. Absent clinical or laboratory data has declined the study population to 198 VLBW infants. Males predominated (53\%). The mean GA was $28.91 \pm 2.67$ weeks and the mean birth weight $1,240.15 \pm 440.76 \mathrm{~g}$. The median NICU stay was 52 days (IQR: 24-82.25 days). Forty-seven (23.7\%) infants died of sepsis.

The proven sepsis rate was $39.9 \%$. Patient demographics (including birth weight and GA) were similar between septic and non-septic infants, but the place of birth and maternal pre-eclampsia status differed $(p=0.046$, and $p=0.026$, respectively). Umbilical catheterisation was the principal sepsis risk factor for blood culture positivity (OR 2.860, 95\% Cl: 1.232-6.639). Infants with proven sepsis exhibited lower day 0 hematocrit and platelet counts, and major changes in hsCRP ratio made within $24 \mathrm{~h}(\mathrm{p}=0.017, \mathrm{p}=0.001$, and $\mathrm{p}=$ 0.022 , respectively).

Outborn infants constituted $47 \%$ of the study population. Although their birth weights and GA were similar to those of inborn infants $(p=0.399$, and $p=0.312)$, outborns were at higher risk of respiratory morbidities requiring frequent surfactant delivery and longer endotracheal intubation and bronchopulmonary dysplasia (BPD), than inborns ( $p=0.013$, $p=0.005$, and $p=0.014$, respectively). Umbilical catheterisation was more often required $(p=0.020)$ and proven sepsis (predominantly Gram-negative bacteria) was more common ( $p=0.045$, and $p=0.033)$. Outborns were at greater risk of proven sepsis and mortality (OR: 1.796, 95\% Cl: $1.011-3.191, p=0.046$; OR: 1.950, 95\% Cl: 1.002-3.794, $p=0.049$ ).
Table I lists mortality by patient demographics and the mortality risks revealed by logistic regression. Non-survivors had lower 5-min Apgar scores $(p<0.001)$ and required frequent invasive procedures (endotracheal surfactant administration, intubation, and venous catheterisation). Birthplace, the Apgar score, endotracheal intubation, necrotising enterocolitis (NEC), surfactant administration and peripheral central venous catheterisation status were evaluated through multivariate regression model. Low 5-min Apgar score was the most significant independent risk factor for sepsis mortality (OR: $13.840,95 \% \mathrm{Cl}$ : 6.384-30.005), followed by NEC and intubation of more than 5 days (OR: 5.410, 95\% Cl: 2.113-13.849, $\mathrm{p}<0.001$ and OR: $10.037,95 \%$ $\mathrm{Cl}: 4.700-21.434, \mathrm{p}<0.001)$. The CBCs and hsCRP levels on days 0 and 1 were subjected to ROC analysis (Table II). Non survivors exhibited abnormal laboratory values (elevations in both parameters) on day $0(p=0.042$, and $p=0.0035$, respectively). The change in hSCRP ratio exhibited the highest area under the curve $(A \cup C)$, sensitivity and specificity: a rise $>6.08$-fold predicted mortality with a sensitivity of $89.36 \%$ and a specificity of $86.09 \%$ (AUC: 0.914 , PPV: $66.7 \%$, NPV: $96.3 \%$ ). An initial WBC $>22.3 \times 10^{9} / \mathrm{L}$ (AUC: $0.611,95 \% \mathrm{Cl}: 0.504-0.718)$ was also associated with mortality. Logistic regression analysis of various combinations of laboratory data showed that the change in hSCRP ratio and initial thrombocytopenia (platelets count $<88 \times 10^{9} / \mathrm{L}$ ) optimally predicted mortality (OR: $27.983,95 \%$ Cl: 9.704-80.697) (Table III).

\section{DISCUSSION}

The 5-min Apgar score, NEC and prolonged endotracheal intubation were independent risk factors for mortality; outborn status affected respiratory outcomes and mortality; the change in hSCRP ratio (calculated from serial hsCRP measurements) sensitively and specifically indicated disease severity; and single laboratory parameters were poorly positively predictive but the combination of the initial platelet count and the change in hsCRP level powerfully predicted septic mortality.

Preterm delivery imposes physical challenges on the newborn. The associated oxidative stress, abnormal gut microbial colonisation and pathogen acquisition impose major risks for sepsis and NEC., ${ }^{3,15,16}$ Low Apgar scores at birth are also associated with an increased risk of bacterial infection. ${ }^{6,17}$ The reduced immunomodulation and impaired defense mechanisms caused by invasive procedures bring along additional risks in preterm infants. ${ }^{1,5,18}$ Low 5-min Apgar score, NEC and prolonged intubation (over 5 days) were three major risk factors for septic mortality, regardless of GA or birth weight. Moreover, non-survivors required frequent antacids (38\%) and umbilical venous catheterisation. Central line-associated bloodstream infections and catheter dwell times over 15 days are known as the most common causes of healthcare-associated infections. ${ }^{19}$ Emphasising the previous statements, prolonged intubation and vascular 
catheterisation should be avoided, and early enteral nutrition progressing to fully enteral feeding prioritised, to reduce the risk of infection during care of VLBW infants.

Birthplace was associated with LOS. Outborns were at increased risk of proven sepsis and mortality (OR: 1.796, 95\%: 1.011-3.191 and OR: 1.950, 95\% Cl: 1.002-3.794). Although the birth weight and GA were similar to those of inborns, outborns were more likely to develop respiratory morbidities (requiring frequent surfactant delivery and longer intubation), BPD and Gram-negative sepsis. Earlier reports described poorer outcomes in terms of intubation requirements, ventilator-induced pneumonia, lung injury and BPD of VLBW neonates not born in level III or IV NICUs; the LOS and mortality rates attained $62 \% .{ }^{20}$ Even when the mortality rate $(23.7 \%)$ was within the previously reported range of 16 to $25 \%,{ }^{2,7}$ birth in level III and IV NICUs delivering special neonatal care might reduce sepsis-related mortality in this population.

The utility of commonly available laboratory data was evaluated, when diagnostic biomarkers may not be assayed in the setting of limited resources. The CBC and CRP level are used by most clinicians to evaluate sepsis. ${ }^{7}$ Unfortunately, their predictive strengths are limited. ${ }^{4,6}$ The CBC alone lacks sensitivity ( 55 to $59 \%$ ) in VLBW infants. ${ }^{2,7}$ Furthermore, the normal CRP range in VLBW infants is unknown given their poor response to inflammatory stimuli. ${ }^{21}$ The level of change in hsCRP ratio was used to identify infants who did not survive sepsis. Non-survivors evidenced significant abnormalities at the very early stage of clinical suspicion, including elevated hsCRP levels and WBCs, compared to survivors. ROC findings confirmed published data; serial hsCRP measurements and calculating the level of difference in the CRP ratio are the best predictors of sepsis mortality. CRP has a half-life of about $19 \mathrm{~h}$ and peaks 24-48 $\mathrm{h}$ after synthesis commences; serial measurements guide therapy. ${ }^{7,21}$ However, the meaningful significance of CRP variations between two-time points remains unclear. ${ }^{2}$ Beltempo et al. found that a difference between two serial CRP measurements of less than $10 \mathrm{mg} / \mathrm{L}$ afforded an NPV of $93-97 \%{ }^{2}$ Different thresholds from 1.5 to $20 \mathrm{mg} / \mathrm{L}$ were associated with wide ranges of sensitivity (48-78\%) and specificity (71-88\%). ${ }^{18,21}$ The change in hsCRP ratio exhibited the highest AUC of the present study; an increase $>6.08$-fold afforded good sensitivity and specificity (AUC: 0.914 ; sensitivity: 89.36, specificity: 86.09 , PPV: $66.7 \%$, NPV: 96.3\%). The difference in the ratio of two serial measurements better predicted mortality than did either measurement alone.

Thrombocytopenia (platelets $<100,000 \mathrm{~mm}^{3}$ ) is a nonspecific late laboratory finding with low discriminatory performance (AUC: 0.60 , sensitivity: $12 \%$ ) in the context of neonatal sepsis. ${ }^{22}$ Moreover, platelet counts vary by GA; agespecific thresholds must be used. ${ }^{13}$ Of our population, $37.5 \%$ had thrombocytopenia, similar to the reported incidences of
10 to $70 \%{ }^{23}$ The initial platelet count did not predict sepsis or mortality; again, thrombocytopenia is a late sign. ${ }^{22}$ The findings has testified the fact that platelet counts must be used in conjunction with other parameters. ${ }^{2,7,22}$ Logistic regression was employed to define the best combination of predictors. A change in hsCRP ratio $>6.08$-fold over $24 \mathrm{~h}$ and a platelet count $<88,000 \mathrm{~mm}^{3}$ on day 0 were associated with a mortality OR of $27.983(95 \% \mathrm{Cl} 9.704-80.697)$. Other previously investigated variables, ${ }^{13,24,25}$ including the WBC, leukopenia, the NLR, the MPV and the PLR, were either poorly or not at all predictive.

This work has several limitations. A retrospective study design is inevitably associated with a risk of bias. Perinatal outborn data (Apgar scores and birth resuscitation requirements) were recorded by physicians commissioned at other centres and may not be completely accurate. It lacked data on hemodynamic status. The diagnostic utilities of procalcitonin or IL-6 levels were not evaluated; because most local NICUs do not measure these markers in their daily practice of neonatal care. The CBC and hsCRP level are universally accepted sepsis workup tools. Their combination can alert neonatal care providers to a sepsis risk.

The strength of this work is that it evaluated LOS in a specific group of newborns, lacking intraventricular haemorrhage and surgical intervention; this eliminated false-positive results.

\section{CONCLUSION}

Low Apgar scores, NEC and prolonged intubation are independent risk factors for mortality of VLBW infants. Birth in level III and IV NICUs featuring special neonatal care, avoidance of prolonged intubation, and timely prediction of fatal sepsis using changes in the hSCRP ratio and platelet count could prevent LOS mortality in limited-resource settings.

\section{ETHICAL APPROVAL:}

The study has been approved by the appropriate ethics committee (Faculty of Medicine, Istinye University, Antalya Medical Park Hospital Ethics Committee, Code: 2019/3; Date: 04.23.2019) and has, therefore, been performed in accordance with the ethical standards laid down in the 1964 Declaration of Helsinki and its later amendments.

\section{PATIENTS' CONSENT:}

This article does not contain any studies with human participants performed by any of the authors. For this type of study, formal consent is not required.

\section{AUTHORS' CONTRIBUTION:}

HO: Designed methods, administration of study for the ethics committee and local authority, participated in data collection, statistical analysis, drafting the article, approval of the final version to be published.

MD: Designed methods, data collection, statistical analysis, drafting the critical revision, approval of the final version to be published. 


\section{CONFLICT OF INTEREST:}

Authors declare no conflict of interest that may influence either the conduct or the presentation of the research.

\section{REFERENCES}

1. Rashwan NI, Hassan MH, Mohey El-Deen ZM, Ahmed AE. Validity of biomarkers in screening for neonatal sepsis - A single center-hospital based study. Pediatr Neonatol 2019; 60(2):149-55.

2. Beltempo M, Viel-Thériault I, Thibeault R, Julien AS, Piedboeuf $B$. C-reactive protein for late-onset sepsis diagnosis in very low birth weight infants. BMC Pediatr 2018; 18(1):16.

3. Adams M, Bassler D. Practice variations and rates of late onset sepsis and necrotizing enterocolitis in very preterm born infants, A review. Transl Pediatr 2019; 8(3):212-26.

4. Puopolo KM, Benitz WE, Zaoutis TE, Committee on fetus and newborn and committee on infectious diseases. Management of neonates born at $\leq 34$ 6/7 weeks' gestation with suspected or proven early onset bacterial sepsis. Pediatrics 2018; 142:20182896.

5. Schüller SS, Kramer BW, Villamor E, Spittler A, Berger A, Levy O. Immunomodulation to prevent or treat neonatal sepsis: past, present, and future. Front Pediatr 2018; 6:199.

6. Shane AL, Sánchez PJ, Stoll BJ. Neonatal sepsis. Lancet 2017; 390:1770-80.

7. Hornik CP, Benjamin DK, Becker KC, Benjamin DK, Li J, Clark RH, et al. Use of the complete blood cell count in late-onset neonatal sepsis. Pediatr Infect Dis J 2012; 31(8):803-7.

8. Hedegaard SS, Wisborg K, Hvas AM. Diagnostic utility of biomarkers for neonatal sepsis-a systematic review. Infect Dis (Lond) 2015; 47(3):117-24.

9. Rhee C, Kadri SS, Danner RL, Suffredini AF, Massaro $A F$, Kitch BT, et al. Diagnosing sepsis is subjective and highly variable: A survey of intensivists using case vignettes. Crit Care 2016; 20:89.

10. Leistner R, Thürnagel S, Schwab F, Piening B, Gastmeier $P$, Geffers $C$. The impact of staffing on central venous catheter-associated bloodstream infections in preterm neonates-results of nation-wide cohort study in Germany. Antimicrob Resist Infect Control 2013; 2(1):11

11. Camacho-Gonzalez A, Spearman PW, Stoll BJ. Neonatal infectious diseases: Evaluation of neonatal sepsis. Pediatr Clin North Am 2013; 60(2):367-89.

12. Polin RA. Committee on Fetus and Newborn. Management of neonates with suspected or proven early-onset bacterial sepsis. Pediatrics 2012; 129:1006-15.
13. Wiedmeier SE, Henry E, Sola-Visner MC, Christensen RD. Platelet reference ranges for neonates, defined using data from over 47,000 patients in a multihospital healthcare system. J Perinatol 2009; 29(2):130-36.

14. Póvoa P, Coelho L, Almeida E, Fernandes A, Mealha R, Moreira $\mathrm{P}$, et al. Pilot study evaluating c-reactive protein levels in the assessment of response to treatment of severe bloodstream infection. Clinical Infectious Diseases. 2005; 40(12):1855-57.

15. Martin A, Faes C, Debevec T, Rytz C, Millet G, Pialoux V. Preterm birth and oxidative stress: Effects of acute physical exercise and hypoxia physiological responses. Redox Biol 2018; 17:315-22.

16. Park JH, Chang YS, Ahn SY, Sung SI, Park WS. Predicting mortality in extremely low birth weight infants: Comparison between gestational age, birth weight, Apgar score, CRIB II score, initial and lowest serum albumin levels. PLoS One 2018; 13(2):0192232.

17. Shah J, Jefferies AL, Yoon EW, Lee SK, Shah PS. Canadian Neonatal Network. Risk factors and outcomes of lateonset bacterial sepsis in preterm neonates born at $<32$ Weeks' gestation. Am J Perinatol 2015; 32(7):675-82.

18. Lai MY, Tsai MH, Lee CW, Chiang MC, Lien R, Fu RH, et al. Characteristics of neonates with culture-proven bloodstream infection who have low levels of C-reactive protein ( $\leqq 10 \mathrm{mg} / \mathrm{L}$ ). BMC Infect Dis 2015; 15:320.

19. Escalante MJ, Ceriani-Cernadas JM, D'Apremont I, Bancalari A, Webb V, Genes L, et al. Late onset sepsis in very low birth weight infants in the south american neocosur network. Pediatr Infect Dis J 2018; 37(10):1022-27.

20. Brasher MI, Patil M, Hagan J, Suresh GK. Mortality and morbidity in outborn extremely low birth weight neonates: A retrospective analysis. J Perinatol 2020; 40(2):337-43.

21. Hofer N, Zacharias E, Muller W, Resch B. An update on the use of C-reactive protein in early-onset neonatal sepsis: Current insights and new tasks. Neonatology 2012; 102(1):25-36

22. Yorulmaz A, Yücel M, Sert S. Diagnostic value of haematological parameters in neonatal sepsis cases. Ortadogu Med J 2018; 10:252-62.

23. Jiang JH, Chiu NC, Huang FY, Kao HA, Hsu CH, Kao H, et al. Neonatal sepsis in the neonatal intensive care unit: characteristics of early versus late onset. J Microbiol Immunol Infect 2004; 37(5):301-6.

24. Sharma D, Farahbakhsh N, Shastri S, Sharma P. BiomarkerS for diagnosis of neonatal sepsis: A literature review. J Matern Fetal Neonatal Med 2018; 31(12):1646-59.

25. Aydemir C, Aydemir H, Kokturk F, Kulah C, Mungan AG. The cut-off levels of procalcitonin and C-reactive protein and the kinetics of mean platelet volume in preterm neonates with sepsis. BMC Pediatr 2018; 18(1):253. 